\title{
Topological Hall and spin Hall effects in disordered skyrmionic textures
}

\author{
Papa Birame Ndiaye, ${ }^{*}$ Collins Ashu Akosa, and Aurélien Manchon \\ King Abdullah University of Science and Technology (KAUST), Physical Science and Engineering Division (PSE), \\ Thuwal 23955-6900, Saudi Arabia
}

(Received 18 September 2016; revised manuscript received 21 December 2016; published 23 February 2017)

\begin{abstract}
We carry out a thorough study of the topological Hall and topological spin Hall effects in disordered skyrmionic systems: the dimensionless (spin) Hall angles are evaluated across the energy-band structure in the multiprobe Landauer-Büttiker formalism and their link to the effective magnetic field emerging from the real-space topology of the spin texture is highlighted. We discuss these results for an optimal skyrmion size and for various sizes of the sample and find that the adiabatic approximation still holds for large skyrmions as well as for nanoskyrmions. Finally, we test the robustness of the topological signals against disorder strength and show that the topological Hall effect is highly sensitive to momentum scattering.
\end{abstract}

DOI: 10.1103/PhysRevB.95.064426

\section{INTRODUCTION}

Since the discovery of the ordinary Hall effect [1] (OHE) in 1879 , closely related phenomena such as the anomalous Hall effect [2] (AHE) and spin Hall effect [3-7] (SHE) have been experimentally reported and their underlying mechanisms theoretically investigated [8,9]. Their occurrence in a broad range of solids and electron gases under different conditions suggests a common denominator which is the conjunction of time-reversal symmetry breaking by either external magnetic field or magnetization and the onset of an effective Lorentz force either driven by external magnetic field or spin-orbit coupling (SOC). In ferromagnetic conductors for instance, where magnetization and SOC are present, AHE generates a transverse charge voltage at opposite edges of the sample [8]. In contrast, in normal metals or semiconductors where only SOC is present, SHE induces a chargeless spin voltage [5-7]. In both $\mathrm{AHE}$ and SHE, SOC induces an effective Lorentz force, related either to a disorder-driven renormalization of the velocity operator or to the band-structure Berry curvature [8,9]. The anomalous velocity arises from the fictitious magnetic field $\mathbf{B}(\mathrm{p})$ that emerges in momentum space.

Interestingly, this emergent magnetic field does not necessarily need to be in momentum space, but can also exist in real space $[10,11]$. It is well known that when electrons flow in a nontrivial magnetic texture, they experience an emergent electromagnetic field $[12,13]$. The emergent electric field $E_{i}^{s}=(s \hbar / 2 e) \mathbf{m} \cdot\left(\partial_{t} \mathbf{m} \times \partial_{i} \mathbf{m}\right)$ produces a spin motive force $[14,15]$, i.e., a time-dependent magnetization $\left(\partial_{t} \mathbf{m} \neq 0\right)$ induces a local spin current $[12,13]$. The emergent magnetic field $\mathbf{B}^{s}=(-s \hbar / 2 e) \mathbf{m} \cdot\left(\partial_{x} \mathbf{m} \times \partial_{y} \mathbf{m}\right) \mathbf{z}$ creates an effective Lorentz force [16] on the flowing electron that changes sign on the two opposite spins, creating a local, spin-dependent $\mathrm{OHE}$. This emergent magnetic field, formed by the solid angle subtended by the magnetic moments of the structure [17], is capable of inducing the transverse motion of electrons like any real magnetic field giving rise to the so-called topological Hall effect [18] (THE) in magnetic textures with nontrivial topology.

\footnotetext{
*papabirame.ndiaye@kaust.edu.sa

†aurelien.manchon@kaust.edu.sa
}

The role of real-space topology has continually increased since the experimental discovery of magnetic skyrmions [19-29], which are topologically nontrivial spin textures [30] in non-centro-symmetric ferromagnetic structures. Skyrmions are in pole position in the racetrack memory search, thanks to prominent features that make them the ultimate bit of information [31]: in contrast with magnetic domain walls, skyrmions are topological defects, localized in space, and present a decent robustness against pinning by magnetic defects, enabling current-driven motion at low current density. Different skyrmion sizes have already been reported in the bulk of B20 compounds or in magnetic multilayers with broken inversion symmetry, mostly below $70 \mathrm{~K}$ but also at room temperature [32]. For instance, a skyrmion diameter of $70 \mathrm{~nm}$ has been obtained in thin-film FeGe [23], as compared to $30 \mathrm{~nm}$ in ultrathin $(\mathrm{Ir} / \mathrm{Co} / \mathrm{Pt})_{10}$ multilayers [29] and $18 \mathrm{~nm}$ for $\mathrm{MnSi}$ [20], down to $2 \mathrm{~nm}$ in $\mathrm{Pd} / \mathrm{Fe} / \mathrm{Ir}(111)$ [33] or even $1 \mathrm{~nm}$ in Fe monolayer deposited on the $\operatorname{Ir}(111)$ surface [24]. These sizes correspond to emergent magnetic fields ranging from 1 to $4000 \mathrm{~T}$. The topological properties of skyrmions ensure that the total flux $\int d^{2} \mathbf{r} B_{z}(\mathbf{r})$ over a sample equals $N \Phi_{0}$ with $N$ the quantized topological winding number or Chern number and $\Phi_{0}=h / e$ the quantum of flux. Recently, the discretized topological Hall effect has been observed [34] in constricted geometry and the emergence of quantum AHE in a skyrmion crystal has been theoretically explored [35]. An intriguing topological spin Hall effect (TSHE) has been obtained numerically in a single skyrmion [36] and vortex [37]. This TSHE displays an atypical energy dependence that contrasts with the one of THE.

In this paper, we focus on the topological electronic transport in ferromagnetic skyrmions, in both clean and disordered regimes. We use a tight-binding model to study charge-spin transport quantities in a ferromagnetic conductor perforated by a single skyrmion or many skyrmions. In particular, we investigate the dimensionless charge and spin Hall angles quantifying the strength of THE and TSHE as a function of the carrier transport energy. We also test the magnitude of these two effects as a function of the skyrmion radius and find that the THE and TSHE reach their saturated values even for small skyrmions. Finally, we inspect the robustness of THE and TSHE as a function of the disorder strength and find that the Hall effect is significantly reduced even when the mean free path is larger than the skyrmion radius. 

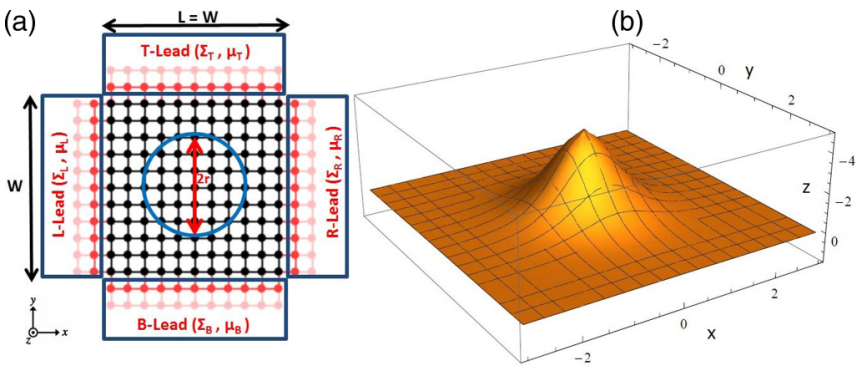

FIG. 1. (a) The four-terminal setup is made up of a central skyrmion scattering region attached to ferromagnetic leads L, T, R, B at chemical potentials $\mu_{L, T, R, B}$. A constant voltage bias is applied between $\mathrm{L}$ and $\mathrm{R}$ while the induced transverse charge-spin voltages are probed in $\mathrm{T}$ and $\mathrm{B}$. (b) The magnetic field emerging from the skyrmionic texture.

The paper is organized as follows: Sec. II presents the theoretical method and offers a general discussion about the charge-spin transport calculation in the tight-binding system. The numerical results for an isolated skyrmion and multiple skyrmions in the clean and disordered regimes are presented and analyzed in Sec. III. Conclusion and perspectives are provided in Sec. IV.

\section{MODEL}

\section{A. Theoretical method}

In this section, we present the system within a suitable framework to quantify the topological Hall effect and the topological spin Hall effect arising from the emergent magnetic field of the skyrmionic texture. The effective field [see Fig. 1(b)] is $B_{z}(\mathbf{r})=\frac{\hbar}{2 e} \mathbf{m} \cdot\left(\partial_{x} \mathbf{m} \times \partial_{y} \mathbf{m}\right)$; the magnetization unit vector defining the spin structure is $\mathbf{m}=\left(\cos \left(m \phi_{x y}+\gamma\right) \sin \theta_{r}, \sin \left(m \phi_{x y}+\gamma\right) \sin \theta_{r}, \cos \theta_{r}\right)$, with $(x, y)=(r \cos \phi, r \sin \phi), r$ being the distance from the origin, center of the skyrmion. $\phi_{x y}$ is the azimuthal angle and $\theta_{r}$ is the radial polar angle, essential to define the skyrmion profile. $m$ is the vorticity, set to 1 for a single skyrmion with $m_{z}=1$ far from the center and -1 at the center whereas $\gamma$ is the helicity that determines the nature of the skyrmion $[30,33]$ : it is hedgehog or Néel-like if $\gamma=0$ and vortex or Bloch-like when $\gamma=\pi / 2$. We model our system, a thin ferromagnetic layer pierced by a single skyrmion $(N=1)$ at its center [see Fig. 1(a)], as a two-dimensional square lattice of size $L \times W a_{0}^{2}$ with $a_{0}$ being the lattice constant, connected to four external semi-infinite ferromagnetic reservoirs to ensure the continuity in magnetization between the central region and the leads. All our considerations are based on steady-state conditions for which the skyrmion is pinned and its dynamics neglected as low current densities are injected.

We calculate the transport properties of interest using the wave-function formulation of the scattering problem as implemented in the software package KWANT [38]. In a single-band tight-binding model, the physical quantities are expressed in the basis of the local atomic sites wave function and the electron Hamiltonian reads

$\hat{H}=\sum_{i} \hat{c}_{i}^{\dagger} \epsilon_{i} \hat{c}_{i}-t \sum_{\langle i, j\rangle}\left(\hat{c}_{i}^{\dagger} \hat{c}_{j}+\right.$ H.c. $)-\Delta \sum_{i} \hat{c}_{i}^{\dagger} \mathbf{m}_{i} \cdot \hat{\boldsymbol{\sigma}} \hat{c}_{i}$

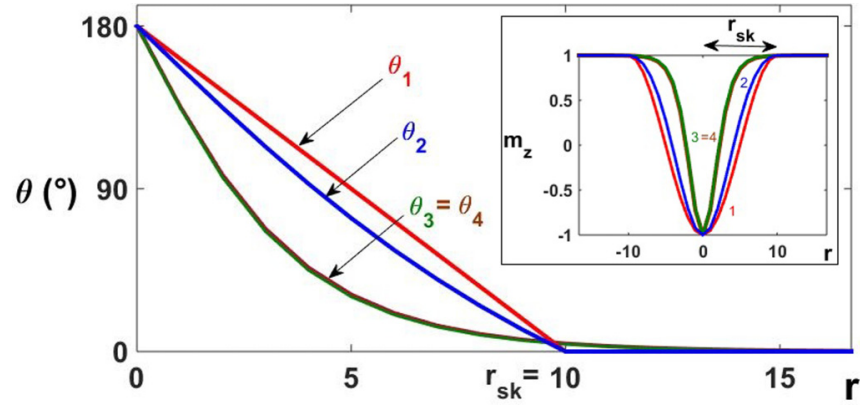

FIG. 2. Different radial distributions of the skyrmion polar angle $\theta_{i}(i=1,2,3,4)$. Inset: The corresponding out-of-plane magnetization spatial profile.

where $\epsilon_{i}$ is the on-site energy, $t$ is the hopping parameter between the neighboring sites $i$ and $j$, and $\Delta$ is the strength of the exchange coupling between the background magnetic texture $\mathbf{m}_{i}$ of the scattering region and the itinerant electron with spin represented by the vector of spin Pauli matrices $\hat{\sigma} \cdot \hat{c}_{i}^{\dagger}=$ $\left(\hat{c}_{i \uparrow}^{\dagger}, \hat{c}_{i \downarrow}^{\dagger}\right)$ is the spinor form of the usual fermionic creation operator at the site $i(\uparrow, \downarrow$ refer to the spin projection along the quantization $z$ axis). The magnetic moment $\mathbf{m}_{i}$ on site $i=$ $\left(i_{x}, i_{y}\right)$, at a distance $r$ from the center of the scattering region, is determined by a single spatial parameter, the skyrmion radius $r_{\text {sk }}$. This radius $r_{\text {sk }}$ is defined in Fig. 2 as the minimal distance for which $\mathbf{m}_{i}\left(\mathrm{r}_{\mathrm{sk}}\right) \simeq 1$ when $\mathbf{m}_{i}(0)=-1$ at the skyrmion core.

To consistently model the skyrmion profile, several numerical approximations for the polar angle $\theta_{r}$ have been used in the literature. Most of these models assume an already relaxed and energetically stable skyrmion state under the competition between different energy terms such as Dzyaloshinskii-Moriya interaction, magnetic anisotropy, exchange, Zeeman coupling, etc. [39]. A detailed treatment of the energy minimization procedure to extract the magnetization texture of a skyrmion remains out of the scope of the present paper, and can be found in Refs. [40,41]. For instance, among the consistent expressions used to model skyrmion profiles, one can find

$$
\begin{gathered}
\left.\theta_{1}\right|_{r \leqslant \mathrm{r}_{\mathrm{sk}}}=\pi\left(1-\frac{r}{\mathrm{r}_{\mathrm{sk}}}\right) \quad \text { and }\left.\theta_{1}\right|_{r>\mathrm{r}_{\mathrm{sk}}}=0, \\
\left.\theta_{2}\right|_{r \leqslant \mathrm{r}_{\mathrm{sk}}}=2 \cos ^{-1}\left(\frac{r^{2}-\mathrm{r}_{\mathrm{sk}}^{2}}{r^{2}+\mathrm{r}_{\mathrm{sk}}^{2}}\right) \quad \text { and }\left.\theta_{2}\right|_{r>\mathrm{r}_{\mathrm{sk}}}=0, \\
\theta_{3}(r)=2 \pi-4 \tan ^{-1} \exp \left(\frac{r}{\mathrm{r}_{\mathrm{sk}} / 4}\right) \\
\theta_{4}(r)=\pi+\sum_{+,-} \sin ^{-1} \tanh \left(\frac{-r \pm c}{\mathrm{r}_{\mathrm{sk}} / 4}\right)
\end{gathered}
$$

The linear profile $\theta_{1}$, Eq. (2), was used in Refs. [11,39], while the Usov ansatz for skyrmion $\theta_{2}$, Eq. (3), was used in Ref. [42]. Both profiles ensure continuous but abrupt boundaries at the skyrmion circumference and therefore present an angular point at $r_{\text {sk }}$, as shown in Fig. 2. On the other hand, the profile $\theta_{3}$, Eq. (4), used in the present paper and the profile $\theta_{4}$, Eq. (5), used in Ref. [33] do not present any abrupt boundary and are 
mathematically equivalent (see Fig. 2). Because the analytical expression $\theta_{4}$ was successfully used to model experimental data [33], we use in this paper the equivalent numerical formulation $\theta_{3}$ for the spin structure of the skyrmion, which is an excellent approximation and fully determined by only one parameter. Notice that as far as transport properties are concerned (i.e., THE and TSHE calculations), the numerical differences between these four profiles are negligible, as long as minimal skyrmion-edge (or skyrmion-skyrmion in a skyrmionic crystal) distances are respected (see below). Finally, neither external magnetic field nor spin-orbit coupling is considered in our investigation. This rules out not only ordinary Hall effect but also the "conventional" anomalous and spin Hall effects. Therefore, any Hall signal computed in this paper arises solely from the topology of the magnetic texture. In realistic samples, where $\mathrm{OHE}$, AHE, and THE coexist, one needs to carefully extract the topological Hall signal using either field dependence [20-22] or inclination-angle dependence [34].

For the coherent charge and spin transport calculation, we apply the Landauer-Büttiker formalism to the four-terminal cross bar device as shown in Fig. 1(a), in which a voltage bias is added between the left lead (L) and the right lead (R), imposing a longitudinal flowing charge current. The induced transverse charge and spin currents are probed using the top lead (T) and the bottom lead (B). Since our objective is to investigate the topological transport emerging from the magnetic texture, we ensure that the shape or geometry of the sample has negligible effects on the physics by adopting large sample sizes (from $64 \times 64$ to $128 \times 128$ sites).

\section{B. Landauer-Büttiker formalism for charge and spin currents}

In our tight-binding model, we define each ferromagnetic lead in Fig. 1 as consisting of two leads allowing only one spin species $\uparrow, \downarrow$ to propagate. The tight-binding Hamiltonian in Eq. (1), with the skyrmion texture, mixes the two spin channels. Therefore, the implementation using KWANT provides directly the spin-resolved transmission coefficients within the standard multiprobe Landauer-Büttiker formalism $[43,44]$. The electric currents $I_{m}^{e}$ in a structure attached to many leads (labeled by $m=\mathrm{L}, \mathrm{T}, \mathrm{R}, \mathrm{B})$ are calculated as

$$
I_{m}^{e}=\frac{e^{2}}{2 \pi \hbar} \sum_{n \neq m, \sigma, \sigma^{\prime}}\left(T_{n m}^{\sigma^{\prime} \sigma} V_{m}-T_{m n}^{\sigma \sigma^{\prime}} V_{n}\right)
$$

where $T_{n m}^{\sigma^{\prime} \sigma}$ is the transmission coefficient for an electron from lead $m$ with spin $\sigma$ to lead $n$ with spin $\sigma^{\prime}$. We note that the vector composed of the four-terminal charge currents is straightforwardly written as a matrix of the transmission coefficients multiplied by the vector of the four lead voltages. The $4 \times 4$ matrix associated with the linear system described by Eq. (6) is obviously singular, because of the total charge current conservation at steady state $\left(T_{m m}^{\sigma^{\prime} \sigma}=-\sum_{n \neq m} T_{m n}^{\sigma^{\prime} \sigma}\right)$. Therefore, we can without loss of generality set one of the voltages $V_{\mathrm{B}}=0$ and write $\left(V_{\mathrm{L}}, V_{\mathrm{T}}, V_{\mathrm{R}}\right)^{\mathrm{T}}=\frac{2 \pi \hbar}{e^{2}} \mathcal{R}\left(I_{\mathrm{L}}^{e}, I_{\mathrm{T}}^{e}, I_{\mathrm{R}}^{e}\right)^{\mathrm{T}}$ where $\mathcal{R}$ is the inverse of the $3 \times 3$ transmission matrix, straightforwardly obtained from Eq. (6). When we enforce a small longitudinal charge bias between lead $\mathrm{L}$ and lead $\mathrm{R}$, i.e., $\mu_{\mathrm{R}}-\mu_{\mathrm{L}}=e \delta V, I_{\mathrm{L}}=-I_{\mathrm{R}}=I$, and $I_{\mathrm{T}}=0$ for the Hall measurements, the terminal voltages are expressed as

$$
\begin{aligned}
& V_{\mathrm{L}}=\left(\mathcal{R}_{11}-\mathcal{R}_{13}\right) \delta V / D, \\
& V_{\mathrm{T}}=\left(\mathcal{R}_{21}-\mathcal{R}_{23}\right) \delta V / D, \\
& V_{\mathrm{R}}=\left(\mathcal{R}_{31}-\mathcal{R}_{33}\right) \delta V / D,
\end{aligned}
$$

and $V_{\mathrm{B}}=0$ with $D=\mathcal{R}_{11}+\mathcal{R}_{33}-\mathcal{R}_{13}-\mathcal{R}_{31}$ and $\delta V=$ $\left(\mu_{\mathrm{L}}-\mu_{\mathrm{R}}\right) / e$ being the imposed voltage bias between the left and right leads. The transverse Hall voltage and the topological Hall angle $\theta_{\mathrm{TH}}$ are readily evaluated as

$$
\theta_{\mathrm{TH}}=\frac{E_{H}}{E_{x}}=\frac{V_{\mathrm{T}}-V_{\mathrm{B}}}{V_{\mathrm{R}}-V_{\mathrm{L}}} .
$$

In order to calculate the spin Hall angle, we first define the quantities

$$
\begin{aligned}
& T_{m n}^{\text {in }}=T_{m n}^{\uparrow \uparrow}+T_{m n}^{\uparrow \downarrow}-T_{m n}^{\downarrow \downarrow}-T_{m n}^{\downarrow \uparrow}, \\
& T_{m n}^{\text {out }}=T_{m n}^{\uparrow \uparrow}+T_{m n}^{\downarrow \uparrow}-T_{m n}^{\downarrow \downarrow}-T_{m n}^{\uparrow \downarrow},
\end{aligned}
$$

quantifying the spin current entering in and going out of the lead $m$. The different terminal spin currents are defined as $[45,46]$

$$
I_{m}^{s}=\frac{e}{4 \pi} \sum_{n \neq m}\left(T_{n m}^{\mathrm{out}} V_{m}-T_{m n}^{\mathrm{in}} V_{n}\right) .
$$

For instance, the spin current in the left lead is $I_{\mathrm{L}}^{s}=$ $\frac{e}{4 \pi}\left(\left[T_{\mathrm{TL}}^{\text {out }}+T_{\mathrm{RL}}^{\text {out }}+T_{\mathrm{BL}}^{\text {out }}\right] V_{\mathrm{L}}-T_{\mathrm{LT}}^{\text {in }} V_{\mathrm{T}}-T_{\mathrm{LR}}^{\text {in }} V_{\mathrm{R}}-T_{\mathrm{LB}}^{\text {in }} V_{\mathrm{B}}\right)$. From the spin and charge currents, we can calculate the topological spin Hall angle (TSH) as

$$
\theta_{\mathrm{TSH}}=\frac{2 e}{\hbar}\left(\frac{I_{\mathrm{T}}^{s}-I_{\mathrm{B}}^{s}}{I_{\mathrm{L}}^{e}-I_{\mathrm{R}}^{e}}\right) .
$$

The topological angles $\theta_{\mathrm{TH}}$ and $\theta_{\mathrm{TSH}}$ give an indication about the effective percentage of charge or spin carriers deflected by the skyrmion spin structure.

\section{RESULTS AND ANALYSIS}

\section{A. Preliminary results for a single skyrmion}

We first benchmark our model by computing $\theta_{\mathrm{TH}}$ and $\theta_{\mathrm{TSH}}$, quantifying the THE and TSHE, respectively, as a function of transport energy $\epsilon_{\mathrm{tr}}$, following Ref. [36]. The system we consider is a typical ferromagnetic metal, described in Eq. (1), such that the splitting of the energy-band structure $\Delta$ is much smaller than the tight-binding bandwidth $8 t$ : the spin-resolved energy bandwidths therefore overlap in some range of the transport energy $\epsilon_{\mathrm{tr}}$. All the energies are normalized to the hopping parameter $t$. The band structure extends from $\epsilon_{\mathrm{tr}}=-4 t-\Delta$ to $4 t+\Delta$, and the associated spin polarization $\mathrm{P}$ is displayed in Fig. 3(a). Half-metallic states $(\mathrm{P}= \pm 1)$ are located at the edges of the band structure, specifically for $\left|\epsilon_{\mathrm{tr}}\right|>4 t-\Delta$. In the range $-4 t+\Delta<\epsilon_{\mathrm{tr}}<$ $4 t-\Delta$, the polarization changes sign continuously, denoting spin mixing. In the rest of the paper, a constant charge bias $\mu_{\mathrm{L}}-\mu_{\mathrm{R}}=10^{-3} t$ is applied to the system. We take $L=W$ unless explicitly specified and all lengths are expressed in units of the lattice parameter $a_{0}$. For different transport energy 

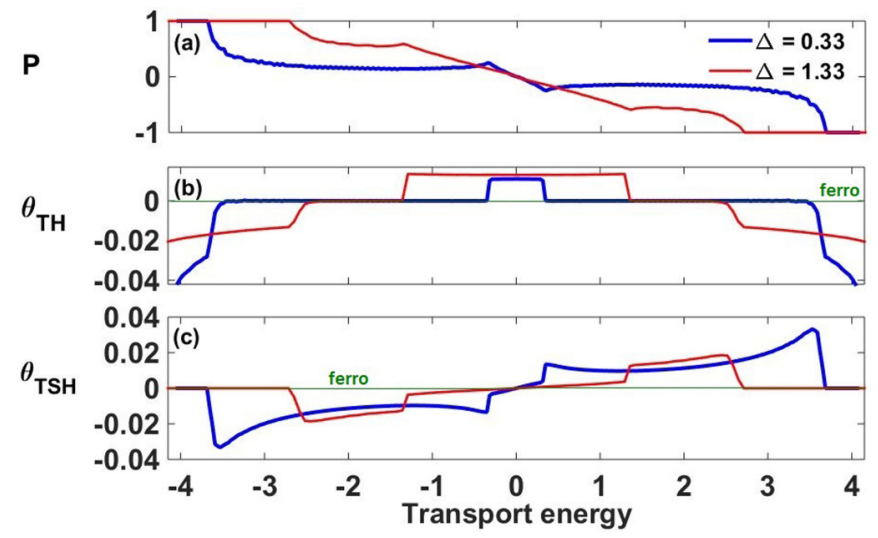

FIG. 3. (a) The spin polarization and (b,c) topological Hall angles for different transport energies $\epsilon_{\mathrm{tr}}$ for a sample size of $L=W=96 a_{0}$ and a skyrmion radius of $10 a_{0}$. The value of the exchange coupling $\Delta$ defines the boundaries and affects the magnitudes of $\theta_{\mathrm{TH}}$ and $\theta_{\mathrm{TSH}}$.

of incoming electrons $\epsilon_{\mathrm{tr}}$, we plot in Figs. 3(b) and 3(c) the topological Hall angle $\theta_{\mathrm{TH}}$ and topological spin Hall angle $\theta_{\mathrm{TSH}}$ for two different values of exchange coupling $\Delta=\frac{1}{3} t$ and $\frac{4}{3} t$. Notice that in the ferromagnetic state, $\theta_{\mathrm{TH}}=\theta_{\mathrm{TSH}}=0$ for all energies and for any exchange coupling, confirming that the origin of the (spin) Hall effects is the skyrmion itself. A global analysis of Fig. 3 shows three main regions, irrespective of the exchange strength.

(1) $\left|\epsilon_{\mathrm{tr}}\right|>4 t-\Delta$; the material is fully spin polarized; $\theta_{\mathrm{TH}}$ is negative and finite whereas $\theta_{\mathrm{TSH}}$ is zero.

(2) $\Delta<\left|\epsilon_{\mathrm{tr}}\right|<4 t-\Delta$; the spin polarization is smaller than 1 and vanishes in most of the region; there $\theta_{\mathrm{TH}}=0$ whereas $\theta_{\text {TSH }}$ is finite and negative.

(3) $\left|\epsilon_{\mathrm{tr}}\right|<\Delta, \theta_{\mathrm{TSH}} \simeq 0$, and $\theta_{\mathrm{TH}}$ is constant and positive.

The dependence of the topological Hall angles $\theta_{\mathrm{T}(\mathrm{S}) \mathrm{H}}$ on transport energy can be understood by considering the spin and carrier type (electron/hole) injected from the ferromagnetic contacts as explained in Ref. [36] [see Fig. 2(d) in Ref. [36]]: for positive bias voltage, electrons are injected from lead $\mathrm{L}$ into lead $\mathrm{R}$ and holes are injected from lead $\mathrm{R}$ into lead $\mathrm{L}$. Under a skyrmion-driven topological Hall effect, a spin-up electron originating from lead $\mathrm{L}$ scatters towards lead $\mathrm{T}$, and by symmetry a spin-down hole originating from lead $\mathrm{R}$ scatters towards lead B. Similarly, a spin-down electron originating from lead L scatters towards lead B, and a spin-up hole originating from lead $\mathrm{R}$ scatters towards lead $\mathrm{T}$. We can now analyze the results displayed in Fig. 3. In region 1, $\left|\epsilon_{\text {tr }}\right|>4 t-\Delta$, the leads are half metallic so that only spin-up is available. Electrons are scattered towards lead $\mathrm{T}$, while holes are scattered towards lead B and as a result only THE survives while TSHE is quenched $\left(\theta_{\mathrm{TSH}}=0\right)$. In region 2 , both spin-up and spin-down electrons (holes) are injected from terminal L (R). Spin-up and spin-down carriers experience a topological spin-dependent force, $F_{\uparrow}=-F_{\downarrow}$, that drags them towards opposite directions. In addition, due to the zero current condition imposed on leads $\mathrm{T}$ and $\mathrm{B}$, the diffusion-driven force reacting to charge imbalance is nontopological and spin independent. Hence, it exerts the same force on spin-up and spin-down, i.e., $-e E_{\mathrm{TH}}=F_{\uparrow}=F_{\downarrow}$. As a consequence, these two conditions are met only when $\theta_{\mathrm{TH}}=0$. In region 3 , spin-down electrons and holes are injected from terminals $\mathrm{L}$ and R, respectively, so that two different types of carriers with the same spin dominate the transport. TSHE is suppressed, and THE becomes finite.

As a final note, we stress that our calculations are performed on large samples and therefore account for a large number of modes. When the calculation is performed in a narrow sample displaying a small number of modes, as in Ref. [36], it results in the manifestation of quantum interferences yielding oscillations of the $\mathrm{T}(\mathrm{S}) \mathrm{HE}$ signal as a function of the energy. Such oscillations are unlikely to be observed in a realistic situation due to decoherence. The large number of modes accounted for in our paper ensures that the computed $\mathrm{T}(\mathrm{S}) \mathrm{HE}$ signals are smooth, free from quantum oscillations, and hence correspond to a more realistic experimental situation.

\section{B. Validity of the adiabatic approximation and the geometrical Hall signals}

The theory of THE has been mostly derived within the adiabatic approximation, i.e., assuming that the flowing spins remain aligned on the local magnetization $[18,30]$. Nonetheless, when the magnetic texture changes abruptly (typically on a distance equivalent to the spin precession length) the itinerant spins start misaligning away from the local magnetization, an effect known as the spin mistracking and responsible for domain-wall resistance and nonadiabatic torque [47,48]. In the present section, we aim at determining whether the result obtained from the adiabatic theory is valid in nanoskyrmions.

To do so, we compute THE and TSHE as a function of the skyrmion size in a sample of width $W=128 a_{0}$. The results are plotted in Fig. 4 for different transport energies: the blue curves represent the THE for $\epsilon_{\mathrm{tr}}=0$ and -3.7 and the red curve represents the TSHE at $\epsilon_{\mathrm{tr}}=-2.0$. The numerical ansatz for the skyrmionic profile described in the previous section is very convenient to avoid spurious effects and unwanted magnetic discontinuity at the edges of the sample. Since we impose the longitudinal bias voltage $\mu_{\mathrm{L}}-\mu_{\mathrm{R}}$, the variation of the $\mathrm{T}(\mathrm{S}) \mathrm{HE}$ reported in Fig. 4 reflects the variation of the induced transverse voltage bias $\mathrm{V}_{T}$.

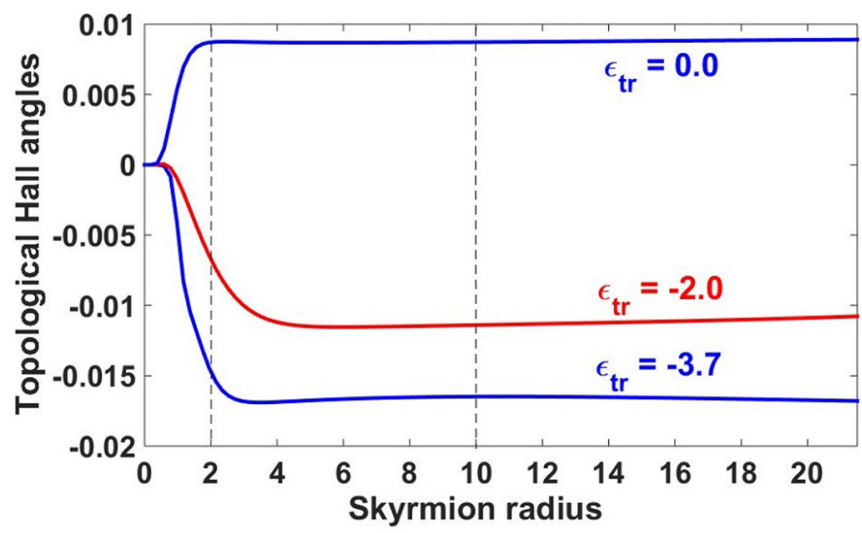

FIG. 4. The nonzero topological Hall angles $\theta_{\mathrm{TH}}$ (blue) and $\theta_{\mathrm{TSH}}$ (red) as a function of the skyrmion radius for three different energies, $\epsilon_{\text {tr }}=-3.7,-2.0$, and 0 . Here, the sample size is $128 \times 128 a_{0}^{2}$ and the exchange coupling is $\Delta=\frac{2 t}{3}$. The vertical lines indicate the minimal and the optimal skyrmion radius we use in the rest of the paper. 
The value $r_{\text {sk }}=0$ corresponds obviously to the absence of the skyrmion, i.e., the homogeneous ferromagnetic state, and does not display any THE or TSHE as seen in Fig. 4. When the radius increases, the system gradually departs from the ferromagnetic state and a single skyrmion is generated so that the topological Hall angles increase from zero to a constant finite value. The radius $\mathrm{r}_{\mathrm{sk}}=a_{0}$ corresponds to a single spin down impurity in the middle of the ferromagnetic state and therefore does not represent a true skyrmion. But above the critical radius of $\mathrm{r}_{\mathrm{sk}}=2 a_{0}$, both THE and TSHE start saturating at a constant value, independent of the skyrmion radius. As a matter of fact, a small skyrmion occupies a narrow region but exhibits a large emergent magnetic field, due to the large magnetization gradient. Hence, although only few electrons experience the emergent magnetic field, they are strongly deflected. On the other hand, a large skyrmion presents a much smaller emergent magnetic field due to its weak magnetization gradient, but occupies a much wider region of space. Therefore, almost all electrons are (weakly) deflected. This balance between strength of emergent field and number of deflected electrons explains the constant value observed in Fig. 4. That is the reason why the theory expresses THE and TSHE as a function of the magnetic flux and not the magnetic field. We conclude that the adiabatic approximation assumed in the conventional theories of THE $[18,30]$ is very robust, even for very small skyrmions. This makes sense since it means that the topological contribution does not change under continuous deformations of the magnetic structure. From now on, we consider a fixed skyrmion size with $\mathrm{r}_{\mathrm{sk}}=\mathrm{r}_{0}=10 a_{0}$.

Finally, we show that our results do not depend on the system size and are therefore independent of the number of modes. Phenomenological reasoning suggests that the longitudinal conductance increases with the sample size while the transverse (topological Hall) conductance is only given by the skyrmion and remains constant as a function of the width. Hence, by applying the appropriate scaling transformation, the resulting $\mathrm{T}(\mathrm{S}) \mathrm{HE}$ curves should all superpose, irrespective of the width of the sample. Figure 5 displays the geometrical

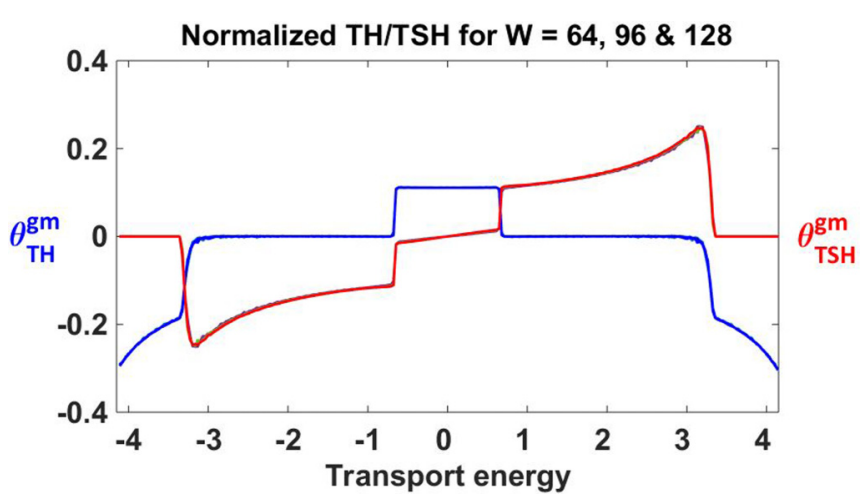

FIG. 5. The normalized $\theta_{\mathrm{TH}}^{\mathrm{gm}}$ (blue) and $\theta_{\mathrm{TSH}}^{\mathrm{gm}}$ (red) as a function of the energy $\epsilon_{\mathrm{tr}}$. The skyrmion radius is $\mathrm{r}_{\mathrm{sk}}=10 a_{0}$. For all widths $W=$ $L=64 a_{0}, 96 a_{0}$, and $128 a_{0}$, the normalized signals are superimposed and give exactly the same value. For reference, the exchange coupling is $\Delta=\frac{2 t}{3}$.
$\mathrm{T}(\mathrm{S}) \mathrm{HE}$, defined

$$
\theta_{\mathrm{T}(\mathrm{S}) \mathrm{H}}^{\mathrm{gm}}=\frac{W}{2 \mathrm{r}_{0}} \theta_{\mathrm{T}(\mathrm{S}) \mathrm{H}}
$$

as a function of the transport energy for various sample sizes, $W=64 a_{0}, 96 a_{0}$, and $128 a_{0}$. As expected, all curves superpose with each other, demonstrating that our results are free from spurious quantum interferences and that the sample boundaries have no impact on our numerical results. Furthermore, Eq. (15) and Fig. 5 indicate that the geometrical Hall angle remains the same for samples of different widths and that the real Hall angle is inversely proportional to the width $W$ of the device. Whereas for large systems the predicted Hall and spin Hall ratios can attain in the best case scenario the value of $4 \%$, the small size samples display an enhanced THE and TSHE that can reach 20\% (not shown-see Ref. [36]). However, this large magnitude of the THE is associated with quantum interferences between the few modes present in the system, which are likely to be smeared out at finite temperature.

\section{Multiple-skyrmion system}

Let us now consider the case of an ordered array of skyrmions. The measurement of T(S)HE with the same setup is performed, the central scattering region being now pierced by $N$ skyrmions instead of an isolated one, like in the case of a skyrmion crystal. Figure 6(a) shows the three positiondependent magnetization components in such a structure: $128 \times 128 a_{0}^{2}$ with a skyrmion number $N=16$ and $\mathrm{r}_{\mathrm{sk}}=10 a_{0}$. In Figs. 6(b) and 6(c) we plot the topological Hall angles $\theta_{\mathrm{TH}}$ and $\theta_{\text {TSH }}$ as a function of the number of skyrmions incorporated in the device. As expected, we obtain a linear dependence of the topological Hall ratios with the skyrmion density.

The magnitude of the topological Hall angles is geometrically enhanced in skyrmion crystals, as the ratio of deflected carriers can reach $50 \%$. Therefore, packing a large number of skyrmions in a large sample produces experimentally detectable THE and TSHE signals with the advantage of the disappearance of the oscillations observed in small size systems. On the other hand, for a given skyrmion radius and

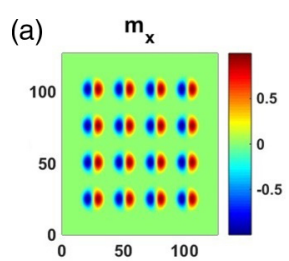

(b)

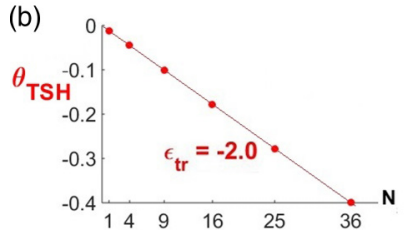

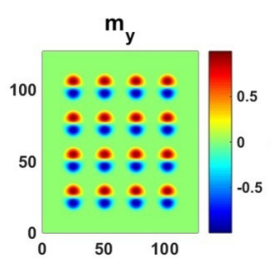

(c)

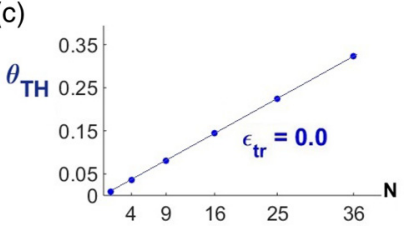

FIG. 6. (a) Spatial variation of the magnetization in a skyrmion crystal $N=16$ for the $128 \times 128 a_{0}^{2}$ sample and a skyrmion radius of $10 a_{0}$. (b,c) The dependence of the (b) THE and (c) TSHE with the number of skyrmions $N$, at the transport energies $\epsilon_{\mathrm{tr}}=-2.0$ and 0.0 , respectively. 


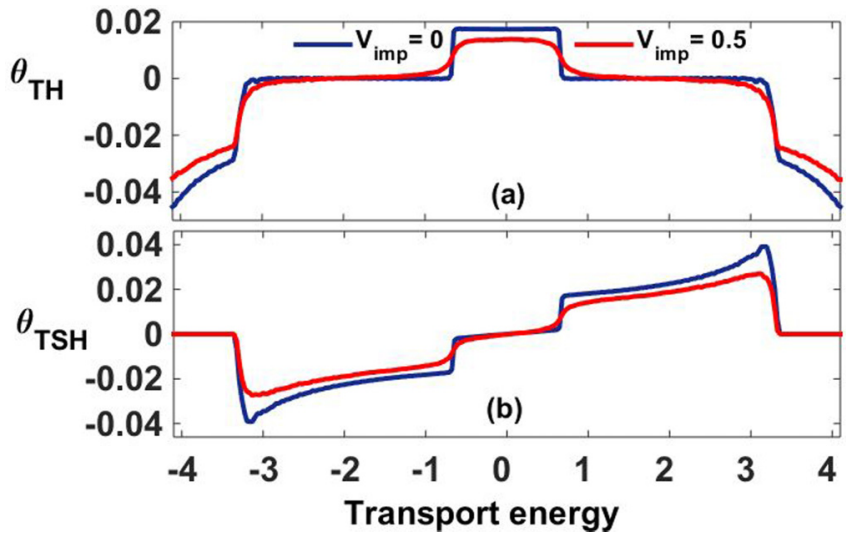

FIG. 7. Energy dependence of (a) $\theta_{\mathrm{TH}}$ and (b) $\theta_{\mathrm{TSH}}$ in the clean limit and for $V_{\text {imp }}=0.5, \Delta=\frac{2 t}{3}$, and $W=L=64 a_{0}$. The signal is reduced and smeared out.

sample size, there is a maximum number of skyrmions that can be incorporated in the configuration described in Fig. 6 $\left(N_{\max }=36\right)$ : the enhancement, though significant, is limited physically by the attainable density of skyrmions in their crystal phase. Minimal skyrmion-skyrmion and skyrmionedge distances have to be respected in our model to avoid spurious size effects, as mentioned above.

\section{Robustness of topological Hall signals}

So far, we have assumed ballistic transport in the clean regime. It has been recently shown that momentum scattering against defects and impurities has a dramatic impact on spin transport in any realistic magnetic textures $[49,50]$. As a matter of fact, since spin transport in magnetic textures presents striking similarities with spin transport in spin-orbit coupled band structures, momentum scattering breaking the coherent spin precession around the local magnetic field results, for instance, in an enhanced nonadiabaticity parameter [51]. Consequently, one expects that impurity scattering is detrimental to the skyrmion induced Hall effects studied above. The aim of this section is to provide some insight on the robustness of $\mathrm{T}(\mathrm{S}) \mathrm{HE}$ in disordered skyrmionic textures.

For simplicity, we will restrain the study of the sensitivity of THE and TSHE to an isolated skyrmion system. The generalization to a multiple-skyrmion system is straightforward. The impurities are numerically introduced in our two-dimensional square lattice by adding a spin-independent random potential $\mathcal{V}_{i}^{\text {si }}$ to the onsite energy $\epsilon_{0}$, such that $\mathcal{V}_{i}^{\text {si }} \in\left[-\frac{V_{\text {imp }}}{2}, \frac{V_{\text {imp }}}{2}\right]$, where $V_{\text {imp }}$ defines the disorder strength. Figures 7 (a) and 7 (b) display the TH and TSH angles in presence (red line) and absence of impurities (blue line): it is shown that weak disorder smears out the edge and boundaries and reduces the magnitude of Hall signals.

For further physical insight, we systematically vary the impurity strength over a wide range $V_{\text {imp }} \in[0,2] t$. In order to quantify the impact of disorder on $\mathrm{T}(\mathrm{S}) \mathrm{HE}$, we first express the impurity strength in terms of its equivalent mean free path $\lambda$. To do so, we calculate the conductance of the two-terminal sample, keeping its width fixed at $W=64 a_{0}$ and varying its length $L$ for different disorder strengths $V_{\text {imp }}$. The

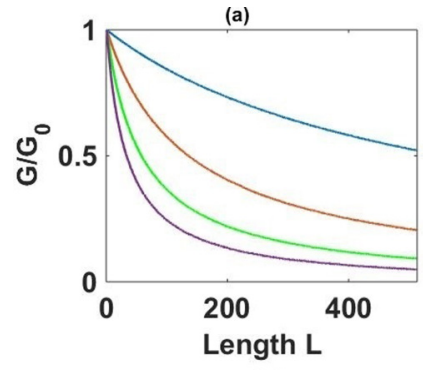

(c)

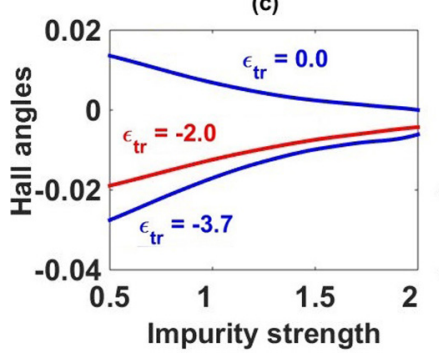

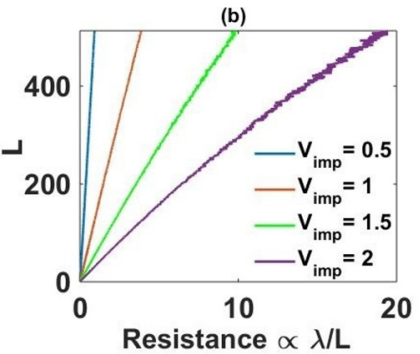

(d)

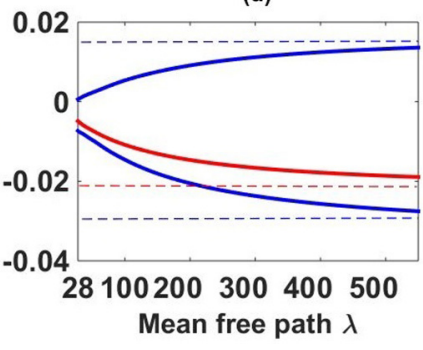

FIG. 8. (a) Normalized conductance of the metallic layer as a function of its length for different impurity strengths. (b) Extraction of the mean free path $\lambda$ for the first transport energy $\epsilon_{\mathrm{tr}}=-3.7$. Note that changing the transport energy modifies the correspondence between $V_{\text {imp }}$ and $\lambda$. (c,d) The nonvanishing $\theta_{\mathrm{TH}}$ (blue) and $\theta_{\mathrm{TSH}}$ (red) for a sample size of $W=64 a_{0}$ and for the three different energies, (c) as a function of impurity strength $V_{\mathrm{imp}}$ and (d) as a function of the equivalent mean free path $\lambda$.

curves of the normalized conductance are shown in Fig. 8(a). We then extract the mean free path corresponding to each disorder strength following the semiclassical formula of the conductance:

$$
G=G_{0} /\left(1+\frac{L}{\lambda}\right)
$$

where $G_{0}=\left(e^{2} / h\right) N$ with $N$ standing for the number of transport modes in the sample. The resistance of the sample and its length follow approximately a linear relationship, the proportionality constant allowing us to extract the effective mean free path of the system, as shown in Fig. 8(b). For $V_{\text {imp }}$ varying from $0.5 t$ to $2 t$, the equivalent mean free path varies from $25 a_{0}$ to $550 a_{0}$. The localization effects are negligible here.

Figures 8(c) and 8(d) display the topological Hall angles as a function of (c) the impurity strength and (d) the equivalent mean free path. The dashed lines in Fig. 8(d) indicate the values of the $\mathrm{T}(\mathrm{S}) \mathrm{HE}$ in the clean limit. These calculations demonstrate clearly that THE and TSHE are very sensitive to disorder. As a matter of fact, although the skyrmion radius is quite small, $\mathrm{r}_{\mathrm{sk}}=10 a_{0}$ in this calculation, the topological Hall angles are reduced by about $50 \%$ for a mean free path about 20 times the skyrmion radius. Notice that in a skyrmion crystal, the magnitude of the topological Hall angle is enhanced but the sensitivity of the signal against impurity scattering is not modified when considering a skyrmion crystal (not shown).

Although these calculations are performed at zero temperature, they suggest that $\mathrm{T}(\mathrm{S}) \mathrm{HE}$ should be quite sensitive to temperature effects. Indeed, increasing the sample temperature promotes low-energy excitations like spin waves and lattice vibrations, and therefore enhances electron-phonon 
and electron-magnon scattering. This naturally leads to the reduction of the mean free path and the increase of the spin-flip scattering rate. Nevertheless, it is remarkable that topological Hall angles of skyrmion crystals are still detectable experimentally at finite temperature [20-22].

\section{DISCUSSION AND CONCLUSION}

The topological properties of electronic transport in skyrmionic textures have been investigated in the clean and disordered regimes. In particular, we showed that the relative strength of the topological Hall and topological spin Hall effects can be discriminated according to the energy of incoming electrons and exchange coupling. The optimal size of the sample and of the skyrmion maximizes the magnitude of the Hall angles, the scale being determined by the geometrical topological Hall angles. Finally, the robustness of these effects with respect to spin-independent impurity scattering is quite weak, as the topological Hall angles are quenched for a mean free path much larger than the skyrmion size.

\section{ACKNOWLEDGMENTS}

This work was supported by the King Abdullah University of Science and Technology through Award No. OSR-CRG URF/1/1693-01 from the Office of Sponsored Research. P.B.N. acknowledges inspiring discussions with A. Abbout, S. Ghosh, and Z. T. Ndiaye.
[1] E. H. Hall, Am. J. Math. 2, 287 (1879).

[2] E. Hall, Philos. Mag. Ser. 5 12, 157 (1881).

[3] M. I. Dyakonov and V. I. Perel, Zh. Eksp. Teor. Fiz. Pis. Red. 13, 657 (1971) [Sov. Phys. JETP Lett. 13, 467 (1971)]; Phys. Lett. A 35, 459 (1971).

[4] J. E. Hirsch, Phys. Rev. Lett. 83, 1834 (1999).

[5] Y. K. Kato, R. C. Myers, A. C. Gossard, and D. D. Awschalom, Science 306, 1910 (2004).

[6] J. Wunderlich, B. Kaestner, J. Sinova, and T. Jungwirth, Phys. Rev. Lett. 94, 047204 (2005).

[7] S. O. Valenzuela and M. Tinkham, Nature (London) 442, 176 (2006).

[8] N. Nagaosa, J. Sinova, S. Onoda, A. H. MacDonald, and N. P. Ong, Rev. Mod. Phys. 82, 1539 (2010).

[9] J. Sinova, S. O. Valenzuela, J. Wunderlich, C. H. Back, and T. Jungwirth, Rev. Mod. Phys. 87, 1213 (2015).

[10] D. Xiao, M.-C. Chang, and Q. Niu, Rev. Mod. Phys. 82, 1959 (2010).

[11] K. Yasuda, R. Wakatsuki, T. Morimoto, R. Yoshimi, A. Tsukazaki, K. S. Takahashi, M. Ezawa, M. Kawasaki, N. Nagaosa, and Y. Tokura, Nat. Phys. 12, 555 (2016).

[12] S. E. Barnes and S. Maekawa, Phys. Rev. Lett. 98, 246601 (2007).

[13] W. M. Saslow, Phys. Rev. B 76, 184434 (2007).

[14] S. A. Yang, G. S. D. Beach, C. Knutson, D. Xiao, Q. Niu, M. Tsoi, and J. L. Erskine, Phys. Rev. Lett. 102, 067201 (2009).

[15] K. Tanabe, D. Chiba, J. Ohe, S. Kasai, H. Kohno, S. E. Barnes, S. Maekawa, K. Kobayashi, and T. Ono, Nat. Commun. 3, 845 (2012).

[16] T. Schulz, R. Ritz, A. Bauer, M. Halder, M. Wagner, C. Franz, C. Pfleiderer, K. Everschor, M. Garst, and A. Rosch, Nat. Phys. 8, 301 (2012).

[17] Y. Taguchi, Y. Oohara, H. Yoshizawa, N. Nagaosa, and Y. Tokura, Science 291, 2573 (2001).

[18] P. Bruno, V. K. Dugaev, and M. Taillefumier, Phys. Rev. Lett. 93, 096806 (2004).

[19] S. Mühlbauer, B. Binz, F. Jonietz, C. Pfleiderer, A. Rosch, Neubauer, R. Georgii, and P. Böni, Science 323, 915 (2009).

[20] A. Neubauer, C. Pfleiderer, B. Binz, A. Rosch, R. Ritz, P. G. Niklowitz, and P. Böni, Phys. Rev. Lett. 102, 186602 (2009).

[21] N. Kanazawa, Y. Onose, T. Arima, D. Okuyama, K. Ohoyama, S. Wakimoto, K. Kakurai, S. Ishiwata, and Y. Tokura, Phys. Rev. Lett. 106, 156603 (2011).
[22] K. Ueda, S. Iguchi, T. Suzuki, S. Ishiwata, Y. Taguchi, and Y. Tokura, Phys. Rev. Lett. 108, 156601 (2012).

[23] X. Z. Yu, Y. Onose, N. Kanazawa, J. H. Park, J. H. Han, Y. Matsui, N. Nagaosa, and Y. Tokura, Nature (London) 465, 901 (2010).

[24] S. Heinze, K. von Bergmann, M. Menzel, J. Brede, A. Kubetzka, R. Wiesendanger, G. Bihlmayer, and S. Blügel, Nat. Phys. 7, 713 (2011).

[25] W. Jiang, P. Upadhyaya, W. Zhang, G. Yu, M. B. Jungfleisch, F. Y. Fradin, J. E. Pearson, Y. Tserkovnyak, K. L. Wang, O. Heinonen, S. G. E. te Velthuis, and A. Hoffmann, Science 349, 283 (2015).

[26] S. Woo, K. Litzius, B. Kruger, M.-Y. Im, L. Caretta, K. Richter, M. Mann, A. Krone, R. M. Reeve, M. Weigand, P. Agrawal, I. Lemesh, M.-A. Mawass, P. Fischer, M. Kläui, and G. S. D. Beach, Nat. Mater. 15, 501 (2016).

[27] O. Boulle, J. Vogel, H. Yang, S. Pizzini, D. de Souza Chaves, A. Locatelli, T. O. Mentes, A. Sala, L. D. Buda-Prejbeanu, O. Klein, M. Belmeguenai, Y. Roussigné, A. Stashkevich, S. M. Chérif, L. Aballe, M. Foerster, M. Chshiev, S. Auffret, I. M. Miron, and G. Gaudin, Nat. Nano. 11, 449 (2016).

[28] G. Chen, A. Mascaraque, A. T. N'Diaye, and A. K. Schmid, Appl. Phys. Lett. 106, 242404 (2015).

[29] C. Moreau-Luchaire, C. Moutafis, N. Reyren, J. Sampaio, C. A F. Vaz, N. Van Horne, K. Bouzehouane, K. Garcia, C. Deranlot, P. Warnicke, P. Wohlhüter, J.-M. George, M. Weigand, J. Raabe, V. Cros, and A. Fert, Nat. Nano. 11, 444 (2016).

[30] N. Nagaosa and Y. Tokura, Nat. Nano. 8, 899 (2013).

[31] A. Fert, V. Cros, and J. Sampaio, Nat. Nano. 8, 152 (2013).

[32] G. Yu, P. Upadhyaya, X. Li, W. Li, S. K. Kim, Y. Fan, K. L. Wong, Y. Tserkovnyak, P. K. Amiri, and K. L. Wang, Nano Lett. 16, 1981 (2016).

[33] N. Romming, A. Kubetzka, C. Hanneken, K. von Bergmann, and R. Wiesendanger, Phys. Rev. Lett. 114, 177203 (2015).

[34] N. Kanazawa, M. Kubota, A. Tsukazaki, Y. Kozuka, K. S. Takahashi, M. Kawasaki, M. Ichikawa, F. Kagawa, and Y. Tokura, Phys. Rev. B 91, 041122(R) (2015).

[35] K. Hamamoto, M. Ezawa, and N. Nagaosa, Phys. Rev. B 92, 115417 (2015).

[36] G. Yin, Y. Liu, Y. Barlas, J. Zang, and R. K. Lake, Phys. Rev. B 92, 024411 (2015).

[37] J-I. Ohe, T. Ohtsuki, and B. Kramer, Phys. Rev. B 75, 245313 (2007). 
[38] C. W. Groth, M. Wimmer, A. R. Akhmerov, and X. Waintal, New J. Phys. 16, 063065 (2014).

[39] N. S. Kiselev, A. N. Bogdanov, R. Schafer, and U. K. Rossler, J. Phys. D 44, 392001 (2011).

[40] A. Bogdanov and A. Hubert, J. Magn. Magn. Mater. 138, 255 (1994).

[41] M. E. Knoester, J. Sinova, and R. A. Duine, Phys. Rev. B 89, 064425 (2014).

[42] J.-V. Kim, Phys. Rev. B 92, 014418 (2015).

[43] M. Büttiker, Phys. Rev. Lett. 57, 1761 (1986).

[44] S. Datta, Electronic Transport in Mesoscopic Systems, 1st ed. (Cambridge University Press, Cambridge, England, 1995).
[45] T. P. Pareek, Phys. Rev. Lett. 92, 076601 (2004).

[46] B. K. Nikolić, L. P. Zârbo, and S. Souma, Phys. Rev. B 72, 075361 (2005).

[47] G. Tatara and H. Kohno, Phys. Rev. Lett. 92, 086601 (2004).

[48] J. Xiao, A. Zangwill, and M. D. Stiles, Phys. Rev. B 73, 054428 (2006).

[49] Z. Yuan, Y. Liu, A. A. Starikov, P. J. Kelly, and A. Brataas, Phys. Rev. Lett. 109, 267201 (2012).

[50] Z. Yuan and P. J. Kelly, Phys. Rev. B 93, 224415 (2016).

[51] C. A. Akosa, W.-S. Kim, A. Bisig, M. Kläui, K.-J. Lee, and A. Manchon, Phys. Rev. B 91, 094411 (2015). 